\title{
The Megapixel EBCCD: \\ a high-resolution imaging tube sensitive to single photons ${ }^{\star}$
}

S. Buontempo ${ }^{1}$, G. Chiodi ${ }^{6}$, I.N. Dalinenko ${ }^{2}$, A. Ereditato ${ }^{1}$, A.V. Ekimov ${ }^{3}$, J.P. Fabre ${ }^{4}$, V.Yu. Fedorov ${ }^{5}$, A. Frenkel ${ }^{6}$, F. Galeazzi ${ }^{6}$, F. Garufi ${ }^{1}$, S.V. Golovkin ${ }^{3}$, V.N. Govorun ${ }^{3}$, N.N. Kalashnikova ${ }^{5}$, V.G. Kossov ${ }^{2}$, E.N. Kozarenko ${ }^{7}$, I.E. Kreslo ${ }^{7}$, L.Yu. Lasovsky ${ }^{2}$, B. Liberti ${ }^{6}$, A.V. Malyarov ${ }^{2}$, G. Martellotti ${ }^{6}$, A.M. Medvedkov ${ }^{3}$, G. Penso ${ }^{6}$, G.I. Vishnevsky ${ }^{2}$, T. Wolff ${ }^{8}$, and A.Zhuk ${ }^{2}$.

\begin{abstract}
A hybrid image-intensifier tube, suitable for extremely low-light imaging, has been tested. This device is based on an Electron-Bombarded CCD chip (EBCCD) with $1024 \times 1024$ sensitive pixels. The tube, which has a photocathode diameter of 40 $\mathrm{mm}$, is gateable and zoomable, with an image magnification varying from 0.62 to 1.3 . The high gain (about 4000 collected electrons per photoelectron at the operational voltage of $15 \mathrm{kV}$ ) and the relatively low noise (180 electrons per pixel at $10 \mathrm{MHz}$ pixelreadout frequency), allows single-photoelectron signals to be separated from noise with a signal-to-noise ratio greater than 10. By applying an appropriate threshold on the signal amplitude, the background can almost be eliminated, with a loss of few percent in single-photoelectron counting. High inner gain, low noise, single-photoelectron sensitivity, and high spatial resolution make the EBCCD imaging tube a unique device, attractive for many applications in high-energy physics, astrophysics, biomedical diagnostics.
\end{abstract}

PACS: 42.79.Ls; 42.79.Pw; 85.60.Gz

Keywords: Image intensifiers; Imaging detectors; Charge-coupled devices; CCD

(Submitted to Nuclear Instruments 8 Methods A)

\footnotetext{
* This work is part of the CERN Research and Development programme RD46.

${ }^{1}$ Università "Federico II" and INFN, Napoli, Italy.

2 Nat. Res. Inst. "Electron", St. Petersburg, Russia.

${ }^{3}$ IHEP, Protvino, Russia.

${ }^{4}$ CERN, Genève, Switzerland.

${ }^{5}$ Inst. of Electron Devices, Moscow, Russia.

6 Università di Roma "La Sapienza" and INFN, Roma, Italy.

7 JINR, Dubna, Russia.

${ }^{8}$ Westfälische Wilhelms-Universität, Münster, Germany.
} 


\section{Introduction}

The possibility of using Charge Coupled Devices (CCDs) for direct detection of charged particles was tested early in their development, both for low-light imaging [1-4] and for high-resolution tracking in high-energy physics [5-7]. In particular CCD chips have been installed in place of the phosphor screen in different types of image intensifiers, to detect the photoelectrons emitted by the photocathode and accelerated by the high voltage applied to the tube.

Conventional CCDs operating in frontside electron-bombarded (EB) mode [1,2,811] have only a reduced active area, due to the presence of the top MOS structures. Moreover they have too low a radiation resistance for most applications, a significant degradation already being measured after an integrated dose of about $10^{6}$ photoelectrons per pixel $[1,8]$.

The development of thinned backside bombarded EBCCDs [1,3, 4, 8, 11-20] was limited by the difficulty in obtaining a uniform sensitivity, and by their relatively short lifetime. In a prototype of an EBCCD tube made by Philips, with $602 \times 288$ pixels [14] illuminated at the very low level of $\sim 2 \times 10^{-4}$ lux (10 photoelectrons per pixel for a frame readout rate of $50 \mathrm{~Hz}$ ), the dark current increased significantly, up to $13 \%$ of the CCD saturation level after 550 hours of operation. A proximity EBCCD tube with a GaAs photocathode, recently developed by Pixel Vision [19,21], is still limited by a short lifetime.

Compared to the best intensified CCD systems based on the second and third generation (with a GaAs photocathode) image-intensifier tubes containing a microchannel plate, the EBCCD imaging tube has a number of advantages: higher spatial resolution, mainly defined by the size of CCD pixel, lower gain fluctuation and gain stability over the instrument lifetime. These EBCCD devices can be used with success in the picosecond streak image technique [22]; in TV cameras for extremely low light level, including cameras with GaAs photocathode; and in high-energy physics for fibre-detector readout $[23,24]$.

Important progress in the production of EBCCD imaging tubes was recently achieved by the joint efforts of Silar ${ }^{1}$, Geosphaera ${ }^{2}$, and $\operatorname{INFN}^{3}[25,26]$. These tubes are now commercially available with $532 \times 290,780 \times 290$, and $1024 \times 1024$ pixels.

In this paper we present the results of a test on the performance of a Megapixel EBCCD zoom tube working in single-photoelectron detection mode, read by a low-noise front-end electronics developed for this purpose.

\section{$2 \quad$ EBCCD tube design and electronics}

The design of the EBCCD chip and the zoom tube was described in detail in a previous paper [26]. Here we repeat only briefly the main points. The Megapixel EBCCD is a full-frame CCD containing $1024 \times 1024$ sensitive pixels, $13.1 \times 13.1 \mu^{2}$ each, which correspond to a total active area of $13.4 \times 13.4 \mathrm{~mm}^{2}$. The chip substrate is thinned down to $\sim 8 \mu \mathrm{m}$, and installed in the tube in such a way to be backside electron-bombarded. The chip is mounted (Fig. 1) into a metal-ceramic housing which is installed in place of the output phosphor, in a first generation, electrostatically focused, zoom tube by

\footnotetext{
${ }^{1}$ Silar, 194223 St. Petersburg, Russian Federation.

${ }^{2}$ Geosphaera Research Centre, P.B. n. 6, 117133 Moscow, Russian Federation.

${ }^{3}$ Istituto Nazionale di Fisica Nucleare, Sezione di Roma, Piazza A. Moro 2, 00185 Roma, Italy.
} 


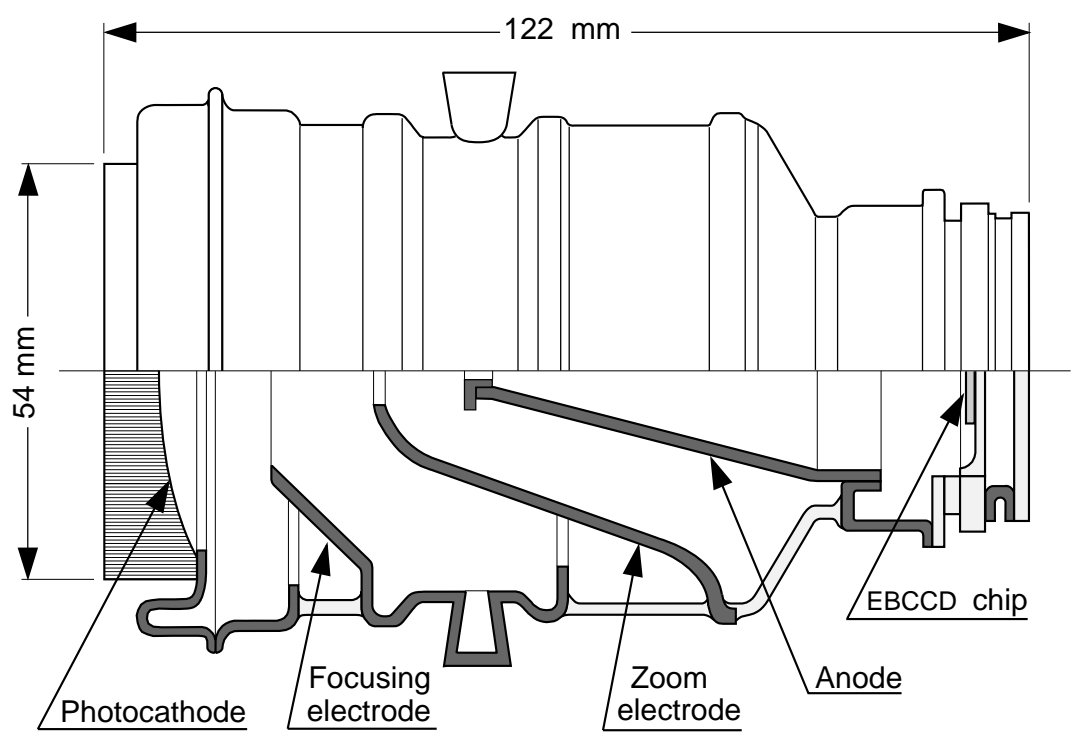

Figure 1: Schematic section of the EBCCD tube.

means of a vacuum flange, fixed to the tube itself by laser welding. The fibre-optic input window has a multialkali photocathode with a useful diameter of $40 \mathrm{~mm}$. The quantum efficiency of the photocathode reaches its maximum value of $10 \%{ }^{4}$ at $500 \mathrm{~nm}$. A negative potential, up to $-15 \mathrm{kV}$, can be applied to the photocathode. The EBCCD tube can be gated by applying pulses of $1.6 \mathrm{kV}$ to the focusing electrode. The length of gate pulses, which is limited only by the electronics capability to drive the gateelectrode capacitance, can be as low as $100 \mathrm{~ns}$. The device has been tested for 2000 hours at the illumination of $2 \times 10^{-2}$ lux. No variations of the tube sensitivity and dark current have been detected. The magnification of the tube can be varied from 0.62 to 1.3 . The geometric distortion of the tube is less than $3 \%$. With an illumination of $2 \times 10^{-4}$ lux and a magnification of 1.3 a spatial resolution of the order of $50 \mathrm{lp} / \mathrm{mm}$ (at $15 \%$ MTF) was measured.

The EBCCD chip has two adjacent active zones of $512 \times 1024$ pixels each and two output registers, one for each zone. A fast, low-noise, compact electronic circuit, based on Altera ${ }^{5}$ MAX 7000 programmable gate arrays, has been designed for the control and readout of the EBCCD. The pixel clock frequency is $10 \mathrm{MHz}$. Low-noise amplifiers and an analog signal processor (correlated double sampling) for noise suppression are used. The readout can be performed through one of the two output registers, or alternatively the two zones of the active area can be shifted in opposite directions and read out in parallel through the two output registers. The total readout time is $104 \mathrm{~ms}$ in the first case, and $52 \mathrm{~ms}$ in the second case.

To reduce the background level given by the dark current of the EBCCD, and the noise associated with it, the EBCCD chip is operated at a temperature around $0{ }^{\circ} \mathrm{C}$ using a Peltier cooler in contact with the metal-ceramic housing of the chip. This reduces the dark current by a factor $\geq 10$ and the total noise by a factor $\sim 2$.

\footnotetext{
${ }^{4}$ Recently a quantum efficiency up to $14 \%$ was reached for the best specimens.

${ }^{5}$ Altera Corporation, 101 Innovation Dr., San Jose, CA 95134, USA.
} 


\section{Experimental results}

\subsection{Experimental method}

Three different tests have been performed on the EBCCD tube: calibration of the readout chain, measurement of the dark-current, and study of the single-photoelectron response. In all of them the tube and its electronics have been placed in a dark box.

To test the single-photoelectron response of the system, a low-intensity diffused light, generated by a Light Emitting Diode (LED), uniformly illuminates the fibreoptic input window of the tube, in such a way that only a few thousand photoelectrons, randomly distributed on the EBCCD area, are detected per frame. The EBCCD tube operates in gated mode, with a gate of $100 \mu \mathrm{s}$, synchronized with the LED pulses. During this time the EBCCD chip integrates the incoming photoelectrons. At the end of the gate the chip is read through one of the two output registers, at a pixel clock rate of $10 \mathrm{MHz}$. After $104 \mathrm{~ms}$ the readout is finished and the entire cycle is repeated many times in one run of measurements. The same readout conditions are used for the dark current and calibration measurements.

The data acquisition system is based on a commercially available framegrabber Matrox ${ }^{6}$ Pulsar connected to the PCI bus of a PC. Analog signals from the EBCCD are digitized by the 8-bit ADC of the Pulsar module.

Before each run of measurements, eight "dark frames" are acquired with no photocathode illumination, and with the high voltage of the tube turned off to prevent the collection of electrons due to thermal emission from the photocathode. These dark frames are then averaged pixel by pixel, and recorded in the $\mathrm{PC}$ memory as a "background frame" which will represent, for a given run, the nominal pedestal position for each pixel. This background frame is then subtracted, pixel by pixel, from each frame acquired and the resulting images are stored for subsequent analysis. This procedure is necessary to compensate for the background non-uniformity across the active area.

\subsection{Calibration and background evaluation}

The calibration constant $(K)$ of the EBCCD, defined as the ratio between the signal $\left(V_{\text {out }}\right)$ in volts at the electronics output and the number $(N)$ of electrons in the potential well of a pixel, has been determined by measuring, with an ADC, the fluctuations of the output signal ${ }^{7}$. The measurement has been made with the tube not illuminated, the signal being in this case due to the dark current of the silicon chip. In fact the total fluctuation of the signal $\left(\sigma_{\mathrm{T}}\right)$ is due to three causes: the fluctuation of the number of electrons present in a potential well, which is assumed to follow a Poisson distribution $\left(\sigma_{\mathrm{N}}=K \sqrt{N}\right)$; the noise $\left(\sigma_{\mathrm{E}}\right)$ of the readout electronics; and the fluctuation $\left(\sigma_{\mathrm{B}}\right)$ introduced by the subtraction of the background frame. Assuming that these three fluctuations can be added quadratically, we obtain: $\sigma_{\mathrm{T}}^{2}=\sigma_{\mathrm{N}}^{2}+\sigma_{\mathrm{E}}^{2}+\sigma_{\mathrm{B}}^{2}$. Taking into account that the background frame is an average on eight dark frames, we obtain: $\sigma_{\mathrm{B}}^{2}=\sigma_{\mathrm{N}}^{2} / 8$. By combining these relations we obtain $K=V_{\text {out }} / N=8\left(\sigma_{\mathrm{T}}^{2}-\sigma_{\mathrm{E}}^{2}\right) /\left(9 V_{\text {out }}\right)$. In the working conditions already described we measured $V_{\text {out }}=6.6 \mathrm{~V} ; \sigma_{\mathrm{T}}=86.0 \mathrm{mV}$. To evaluate $\sigma_{\mathrm{E}}$ we follow the same procedure but shifting the CCD lines into the output register not connected to the readout, so that no electrons from potential wells

\footnotetext{
${ }^{6}$ Matrox Graphics Inc., 1055 St. Regis blvd. Dorval H9P 2T4, Quebec, Canada.

${ }^{7}$ An offset has been applied to the output signal, so that the ADC dynamics (256 channels, $7.8 \mathrm{mV} /$ channel) allows the output fluctuations to be measured with a good accuracy.
} 

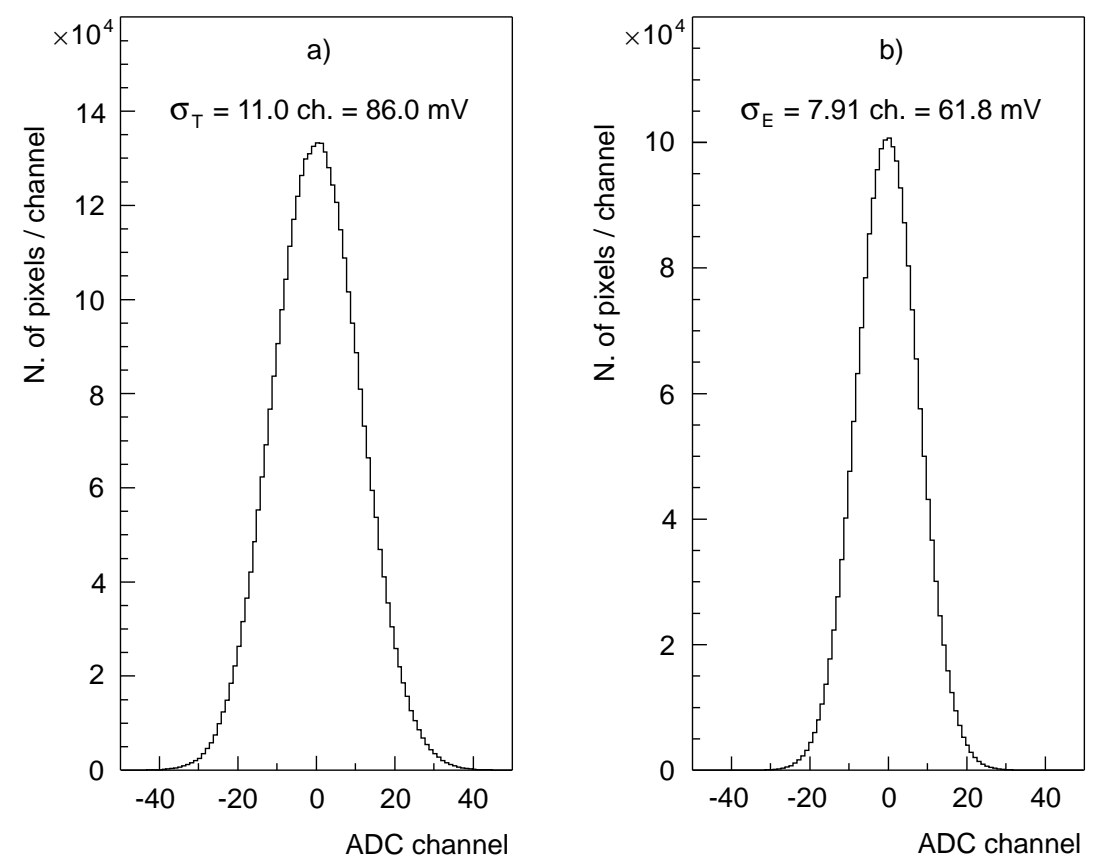

Figure 2: Fluctuations of the output signal around its mean value, owing to a) dark current fluctuations and electronic noise, and b) electronic noise only.

are fed into the electronic chain. The measured fluctuation of the output voltage is therefore due to the electronic noise only. We found $\sigma_{\mathrm{E}}=61.8 \mathrm{mV}$. In Fig. 2 we show the distribution of the total fluctuations and of the electronic noise, as measured by the ADC.

By combining all these measurements we obtain $K=0.48 \mathrm{mV} /$ electron and $N \simeq$ 13800 . We estimated the error on these quantities to be $\pm 10 \%$. The value of $N$ we have obtained is an average on all the CCD pixels. If the same procedure is applied to restricted CCD zones, we found that $N$ varies smoothly across the CCD area by $\pm 5 \%$ of its mean value. Taking into account the obtained value of $K$, the measured values of $\sigma_{\mathrm{T}}$ and $\sigma_{\mathrm{E}}$ are equivalent respectively to $\sim 180$ and $\sim 130$ electrons per pixel. The background level, and thus its fluctuation $\sigma_{\mathrm{N}}^{2}$, can be reduced by a factor two by reading the EBCCD in $52 \mathrm{~ms}$ through the two output registers. A further reduction can be obtained by lowering the operational temperature of the chip with a more efficient Peltier cooler. For example, at $-20^{\circ} \mathrm{C}$ we expect a reduction of this level by a factor $\sim 5$.

\subsection{Single-photoelectron detection}

In order to test the EBCCD capability of detecting single photoelectrons, the tube was uniformly illuminated as described in Section 3.1. The photoelectrons are accelerated in the tube and may strike the EBCCD pixels in random positions. The electrons liberated in the silicon chip are collected in a cluster of a few pixels located around the impact point of the photoelectron. The illumination was kept at a level such that the probability of coalescent clusters was relatively small. A preliminary rough check of the uniformity of the tube response to single photoelectrons, showed that its gain varies by about $\pm 20 \%$ across the $C C D$ area. In the following we have considered only 

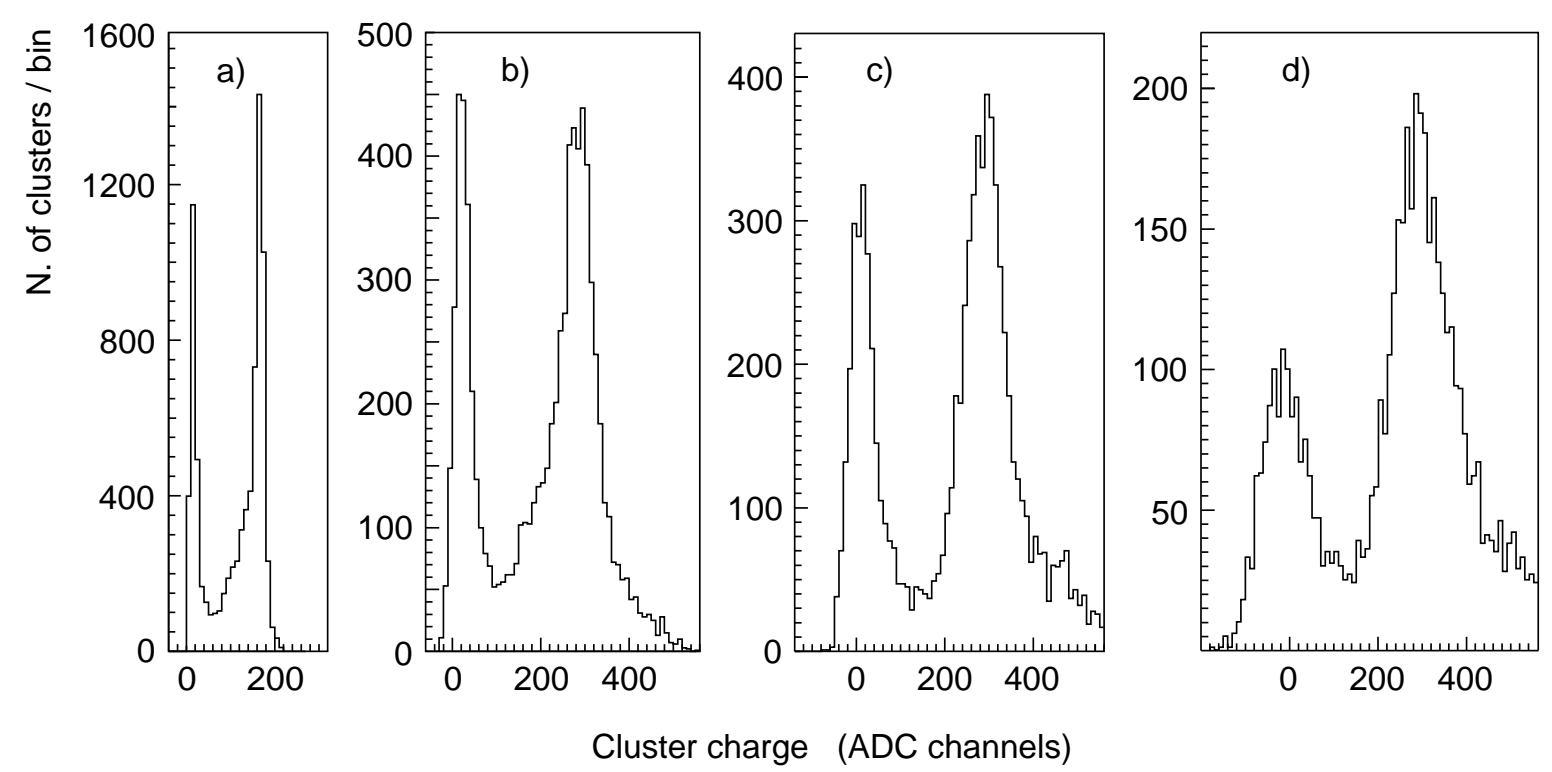

Figure 3: a) Distribution of the charge relative to the local maxima, as defined in the first analysis method; b), c), and d) Distribution of the total charge contained respectively in clusters of three, five and nine pixels, centred on the local maxima. In b) the three considered pixels are aligned along the CCD lines. In all these distributions the single-photoelectron peak is very well separated from the background.

a restricted high-gain region on the CCD, of $100 \times 150$ pixels.

The analysis of the single-photoelectron signal was performed with two methods. In the first method we define a "local maximum" as a pixel having its output signal higher than that of all the eight neighbouring pixels. To avoid coalescent clusters due to close incident photoelectrons, we imposed an "isolation" condition by requiring that no other local maxima are present in the region of $5 \times 5$ pixels centred on the considered local maximum. With these criteria many local maxima have been selected. In Fig. 3a-d we report the distributions of the total charge contained respectively in the local maximum, and in a cluster of three, five or nine pixels comprising the local maximum and the adjacent pixels. In all these distributions the single-photoelectron peak is very well separated from the background peak.

In order to measure the average size of the single-photoelectron clusters, we first selected all the nine pixel clusters having a total charge larger than 110 ADC channels (Fig. 3d). Then we superimposed the 25 pixels centred on the local maxima, by putting their centroid ${ }^{8}$ at the origin of an $(x, y)$ plane. The resulting charge distribution, reported in Fig. 4, appears to be wider and slightly asymmetrical in the $y$ direction (CCD lines), which is parallel to the output registers, compared to the $x$ direction. This effect, which has not yet been fully investigated, is likely due to the response of the amplifier system at high clock frequency.

From the charge distribution reported in Fig. 4 we deduce a spatial resolution of the system of $\sim 13 \mu \mathrm{m}$ (FWHM) in the $x$ direction and of $\sim 26 \mu \mathrm{m}$ in the $y$ direction. More than $\sim 40 \%$ of the total charge is contained in one pixel, and $\sim 80 \%$ is contained

\footnotetext{
${ }^{8}$ The coordinates of the centroid of a cluster of pixels are the average coordinates of all the pixels, weighted with the pixel charge.
} 


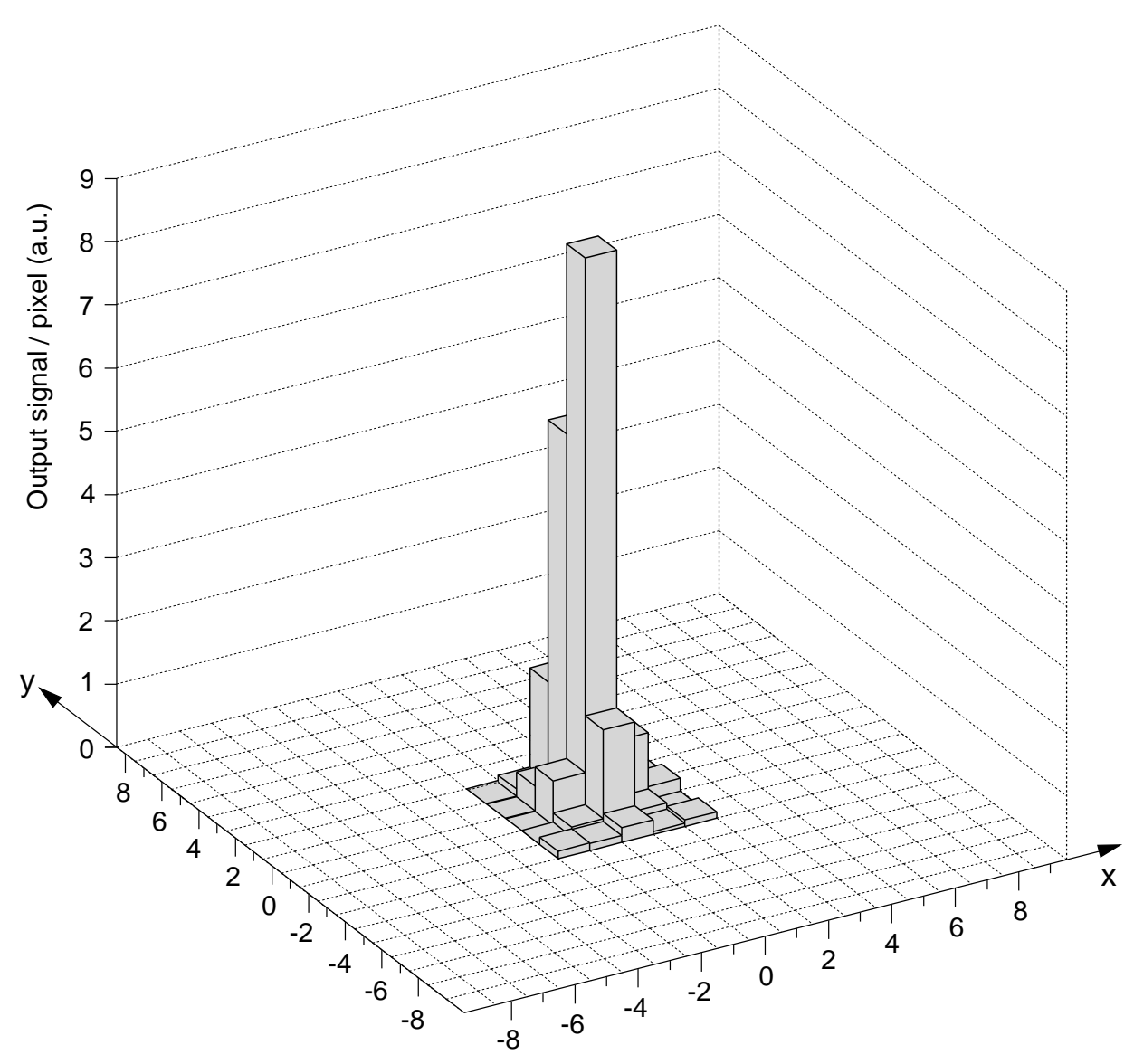

Figure 4: Average distribution, on the CCD plane, of the charge liberated by a single photoelectron. The $y$ axis is parallel to the CCD lines. The units on the two axes are equivalent to a pixel side $(13.1 \mu \mathrm{m})$. The plot has been obtained by superposing at the origin of the $(x, y)$ plane, the barycentre of the charges delivered by many photoelectrons.

in three pixels aligned along the $y$ direction.

The signal-to-noise ratio, defined as the average pulse height of the photoelectron peak divided by the width ${ }^{9}$ of the background peak, is equal to 20, 14, 10, 5 for, respectively, the local maximum pixel and the three-, five- and nine-pixel clusters (Fig. 3a-d).

From Figs. $3 \mathrm{~d}$ and 4 we deduce that the total charge delivered by a photoelectron in the selected high-gain region, is equivalent to $\sim 320$ ADC channels, which correspond to $\sim 5000$ electrons per photoelectron. If we consider the full CCD area, we obtain an average gain of $\sim 4000$ electrons per photoelectron, in good agreement with the value measured [26] with a different method on another similar tube.

The single photoelectron response has also been studied with a second method. In this method we select only those pixels having a pulse height larger than the average background by $20 \mathrm{ADC}$ channels $\simeq 2 \sigma_{\mathrm{T}}$. A cluster is defined as a group of adjacent pixels above this threshold. This method is more selective against background clusters than the first method. Because no "isolation" condition is imposed, it accepts also clusters due to more than one coalescent photoelectrons. The total pulse height of a

\footnotetext{
${ }^{9}$ The width is calculated as the FWHM divided by 2.35 .
} 


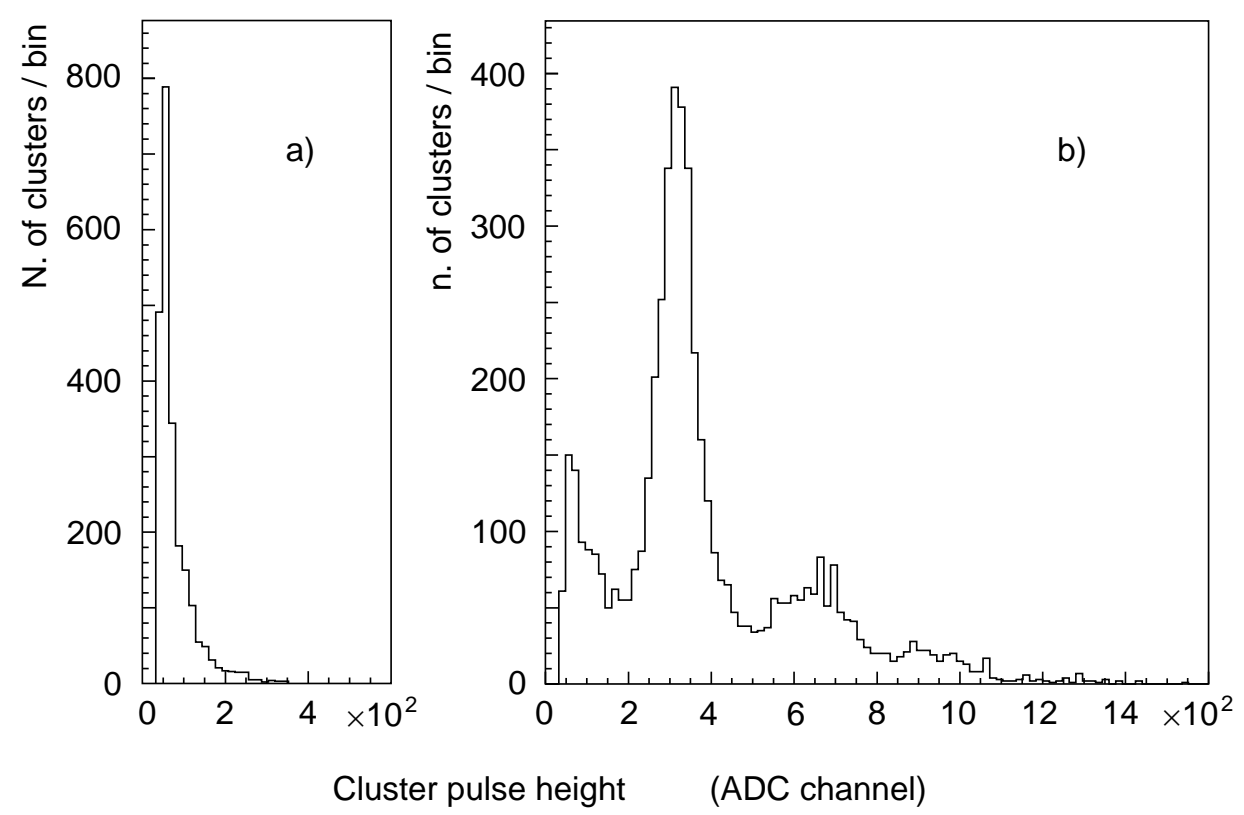

Figure 5: Distribution of the total charge contained in the clusters, as defined in the second analysis method. a) Dark frames; b) illuminated frames. The peaks due to 1 , 2 and 3 photoelectrons are seen.

cluster is, as usual, defined as the sum of the pulse height of the pixels.

The same procedure has been applied, for comparison, also to dark frames. The measured distribution of the total cluster pulse height is reported in Fig. 5a for dark frames, and in Fig. 5b for illuminated frames. In Fig. 5b the tail of the background distribution is observed, together with the one-, two- and even three-photoelectron peaks. The distance between the peaks corresponding to one and two photoelectrons is $\sim 320$ ADC channels, in agreement with the first method.

The widths of the signal peaks (Figs. $3 \mathrm{~d}$ and $5 \mathrm{~b}$ ) are significantly larger than we expect, taking into account the electronic noise, the background fluctuations, and the fluctuation on the number of electron-hole pairs created in the silicon chip, the last of these being negligible with respect to the others, also because of the Fano factor [27]. This effect is presumably related to the non-uniformity of the dead layer on the entrance surface of the CCD, which may cause a variation in the number of electrons liberated in the active part of the chip. Also the collection efficiency of these electrons could be slightly dependent on the impact position of the photoelectron, so giving rise to an increase of the signal fluctuations.

\section{Conclusions}

We have investigated the response to single photoelectrons of a Megapixel EBCCD imaging tube with its readout electronics. We found an average tube gain of about 4000 electrons per photoelectron, at a voltage of $15 \mathrm{kV}$. The electronic and the total noise are equivalent respectively to $\sim 130$ and $\sim 180$ electrons per pixel, at $10 \mathrm{MHz}$ pixel-readout frequency. A signal-to-noise ratio larger than 10 was obtained. With an appropriate threshold on the signal pulse height, the noise can almost be eliminated with a loss of few percent in single-photoelectron counting. The spatial resolution 
ranges between $13 \mu \mathrm{m}$ and $26 \mu \mathrm{m}$ on the CCD plane depending on the considered direction on that plane.

The features of the EBCCD tube make this device unique for high resolution imaging at extremely low light level. The tube described in this paper has been successfully used [28] in the readout of a microvertex detector, to observe neutrino interactions at the CERN Super Proton Synchrotron. Other EBCCD tubes, based on this Megapixel EBCCD chip, are under study. Among them we can cite a magnetic focused Megapixel EBCCD used as the readout system of an optoelectronic delay tube [29], for high-rate experiments in high-energy physics, and a large EBCCD tube with an 80-mm-diameter photocathode and demagnification of 5 , for high-energy physics and biomedical applications.

\section{References}

[1] J. L. Lowrance et al., Proc. SPIE 172 (1979) 232.

[2] J. P. Choisser, Opt. Eng. 16 (1977) 262.

[3] J. L. Lowrance et al., Adv. Electron. Electron. Phys. 52 (1979) 441.

[4] R. G. Hier et al., Adv. Electron. Electron. Phys. 52 (1979) 463.

[5] C. Damerell, Proc. Workshop on Silicon Detectors for High Energy Physics, Fermilab, October 1981, ed. T.Ferbel, p. 33.

[6] A. Bross, Nucl. Instr. and Meth. 201 (1982) 391.

[7] V. N. Govorun et al., IHEP report IHEP 83-136, 1983.

[8] J. C. Cheng et al., J. Appl. Phys. 49 (1978) 5421.

[9] P. T. E. Roberts et al., Ultramicroscopy 8 (1982) 385.

[10] P. Everett et al., Opt. Eng. 24 (1985) 360.

[11] G. R. Carruthers et al., Adv. Electron. Electron. Phys. 74 (1988) 181.

[12] P. Zucchino et al., Proc. SPIE 290 (1981) 174.

[13] L. Bergonzi et al., Adv. Electron. Electron. Physics, 74 (1988) 165.

[14] J. C. Richard et al., Nucl. Instr. and Meth. A315 (1992) 368.

[15] R. G. Hier et al., Proc. SPIE 363 (1982) 57.

[16] R. G. Hier et al., Adv. Electron. Electron. Phys. 74 (1988) 55.

[17] J. C. Richard and M. Lemonier, Proc. Int. Topical Meeting on Image Detection and Quality, (1986) p. 13.

[18] R. Schaefer et al., Proc. SPIE 1447 (1991) 165.

[19] W. Enloe et al., Proc. SPIE 1655 (1992) 41.

[20] I. Debusschere et al., European Space Agency report ESA SP-356, 1992, p. 81. 
[21] G. M. Williams et al., Proc. SPIE 2551 (1995) 208.

[22] G. I. Bryukhnevich et al., Proc. SPIE 1655 (1992) 94.

[23] C. Cianfarani et al., Nucl. Instr. and Meth. A339 (1994) 449.

[24] P. Annis et al., Nucl. Phys. B (Proc. Supp.) 54B (1997) 86.

[25] I. N. Dalinenko et al., Proc. SPIE 2551 (1995) 197.

[26] S. V. Golovkin et al., Proc. SPIE 2551 (1995) 118.

[27] U. Fano, Phys. Rev. 70 (1946) 44; ibid. 72 (1947) 26;

W. Van Roosbroek, Phys. Rev. 139 (1965) A 1702.

[28] R. Van Dantzig et al., RD46 Proposal, CERN/LHCC 95-7, P60/LDRB (1995);

P. Annis et al. Nucl. Phys. B (Proc. Supp.) 61B (1998) 390.

[29] A.G. Berkovski et al., Nucl. Instr. and Meth. A 380 (1996) 537. 